\title{
To accept or not to accept: RED's way
}

\author{
Yoram Reich
}

Published online: 19 September 2010

(C) Springer-Verlag London Limited 2010

There is virtually no paper that is accepted after the review process without revision and very few that do so with minor revisions. On an average, $30 \%$ of the papers submitted to RED are not accepted immediately by the editors (chief or area) being out of scope or far from being at the level of journal quality; 50\% end up being not accepted after the review process; and a mere $20 \%$ are accepted after at least one revision.

Clearly, we wish to publish the best papers in engineering design research; however, doing justice to this topic requires more than an editorial. Consequently, I chose the opposite approach of briefly discussing how to improve the chances of acceptance from RED. In the spirit of Pareto, I will concentrate on few items that are responsible for the majority of nonacceptance.

Each paper submitted to RED passes a review process described in a previous editorial (Reich 2010a). The paper is screened by the Editor-in-Chief and subsequently by an area editor that distributes the paper to reviewers. Altogether, the review process needs to serve two primary goals:

1. Managing the journal mission

Journal scope. A new submission must fit the scope of the journal. Failing to do so is the primary cause for rejection. Most papers that are not accepted immediately simply do not fit the journal scope. A paper that does not have a general statement about engineering design cannot be considered for publication in RED. A paper that deals with a particular product design even

Y. Reich (ه)

Tel Aviv, Israel

e-mail: yoram@eng.tau.ac.il if it requires the use of sophisticated tools is probably outside the scope of RED. A paper whose focus is on the classic use of CAD tools or issues related to such tools is also outside the scope of RED. In most cases, it is easy to decide whether a paper fits the journal scope; in such cases, it is easy to locate other journals that are most appropriate for that paper. Authors should look carefully at the published journal scope and decide whether RED is their best choice before submitting a paper.

2. Maintaining and improving the journal quality

a. Scientific soundness. The lack of soundness is the second reason for not accepting a new submission. Roughly, this means that the paper does not state clearly its value to its readers and demonstrate this value convincingly through acceptable scientific means. Claims that are not substantiated in a way that matches their worldview would lead to rejection. For example, in the praxis worldview, a claim that a method assists designers must be demonstrated on a real case with real designers or at least on a complex case in some limited design setting, while in the scientism worldview, methods derivations should be coherent (Reich 2010b). The demonstration of claims and the paper as a whole are always evaluated subjectively, and indeed, we get conflicting reviews on a regular basis. This is the reason for asking three reviewers to review papers and placing the final judgment in the editors' hands. Sometimes it is a tough decision; nevertheless, authors could contest such decisions and additional reviews could be sought if justified. Another failing issue is a literature review that is not critical but only mentions claims made by the 
authors of cited papers without comments of the present authors; this could be viewed as being sloppy and weakening the basis for justifying the present work.

b. Projected value. A new submission should have one or more of the following properties: it might be considered as a seminal or a ground-breaking paper with future value to the discipline, or it is likely to be cited well in the future-a signal of its quality and a help for improving the impact factor of the journal. Both these could be accepted as quality indicators or projected value to the design community. In contrast, a small increment of previous work, especially if the old work has not been cited ever, is not likely to be accepted. This means that even sound papers that deal with some aspect of design research might not be published in RED if their added value to the community cannot be demonstrated. It is rare, however, that papers are rejected by one editor solely based on their lack of projected value. Most often, a second editor is involved in such decision or the paper goes through the review process leaving reviewers to comment on the paper value. It is a prime task of authors to articulate clearly and upfront the value of their paper.

c. A new submission should create continuity for journal readers by referring and reinterpreting previous work published in the journal that is relevant to the present paper. This should not be interpreted as creating an artificial boost to the impact factor by increasing journal self-citations ${ }^{1}$ but asking that authors be responsible to know prior published work in the journal they are publishing and place their new work in that context. In many cases, the review suggests papers appearing in other journals that are recommended as additional references to authors.

Another issue that could lead to paper rejection is presentation quality. Although it could be improved, poor presentation might prevent reviewers from understanding and appreciating the quality and value of a paper. A new submission should ease its review and reading; think of it as the user interface of a new product that needs to be as friendly as possible. The paper should be written in good language, be logical and structured well, and its presentation should be appealing. Good presentation also invites reviewers and readers to read early and could lead to quicker review process and subsequent referencing by other authors.

I hope that these comments will help authors to pay more attention to initial submissions and, consequently, result in an increased number of quality submissions and accepted papers.

\section{References}

Reich Y (2010a) Editorial, The redesign of Redesign Research in Engineering Design 21(2):65-68

Reich Y (2010b) Editorial, My method is better! 21(3):137-142

\footnotetext{
${ }^{1}$ RED has the least number of self-citations of all design research related journals.
} 